\section{A comparison of five methods of}

\section{illusion measurement}

\author{
STANLEY COREN* \\ The Graduate Faculty of the New School for Social Research \\ New York, New York 10011 \\ and \\ JOAN S. GIRGUS \\ The City College of the City University of New York \\ New York, New York 10031
}

The relative efficiency of five techniques of illusion measurement were tested on parametric variations of the Mueller-Lyer and Ebbinghaus figures. The methods of average error, reproduction, and selection from a graded series all showed significant effects of configurational variations. The subjective scaling techniques each failed to measure adequately changes in illusion magnitude for one illusion configuration. The suitability of the tested measures for group administration is also discussed.

Ever since the turn of the century, when Judd (1899, 1902, 1905), Lewis $(1908,1909)$, and Seashore and his associates (Seashore \& Williams, 1900, 1902; Seashore, Carter, Farnum, \& Sies, 1908) were beginning the parametric quantitative study of visual geometric illusions, the method of average error (sometimes called the method of adjustment) has been the most frequently utilized method of measurement. In this method, $S$ sets an adjustable figural component to match a corresponding component distorted by the illusion. The popularity of this method of illusion measurement has persisted over the last 70 years, although many other measures of illusion magnitude have appeared in the literature since these early investigations.

A random sampling of 60 recent illusion articles reveals an interesting distribution of measurement techniques. These articles were selected from the American Journal of Psychology, Journal of Experimental Psychology, Perception \& Psychophysics, Perceptual and Motor Skills, Psychonomic Science, Quarterly Journal of Experimental Psychology, and Scandinavian Journal of Psychology, between 1967 and the first three months of 1972. The method of average error still dominates, as is evidenced by its use in $\mathbf{4 5 \%}$ of the sampled articles. All of the experiments in the sample that utilized this technique tested Ss individually. This method has been used to measure illusions of extent (Burnham, 1968; Coren, 1970b), direction (Coren, 1969; Coren \& Girgus, 1972), and contour or curvature (Coren, 1970a). A typical example of its use is found in Festinger, White, \& Allyn (1968),

* Mailin address: 66 West 12 th Street, New York, New York 10011. among others. In this situation, the apparently longer half of a Mueller-Lyer figure is made adjustable in length. S's task is to set this adjustable portion of the figure until it appears to be equal in length to the apparently shorter half of the configuration. One persistent problem with the method of average error is the difficulty in constructing apparatus that permits variation of certain stimulus dimensions, such as curvature or shape.

The second most prevalent form of measurement in the sample is based upon S's ability to reproduce the perceived illusory distortion either by drawing it or by marking off an appropriate extent on a comparison figure. This method was employed in $12 \%$ of the sampled experiments. A typical example of its use in an illusion of extent is found in Erlebacher and Sekuler (1969), who required Ss to mark off the apparent length of the shaft of a projected Mueller-Lyer figure on a line provided on a sheet of paper in front of them. Pressey and Sweeney (1969) have adapted this method to measure an illusion of direction. One of the advantages of the method of reproduction is that it requires little apparatus. In addition, it may be used for group presentations.

The methods of constant stimuli and paired comparison account for $10 \%$ of the studies surveyed. These methods usually involve successive comparisons of pairs of figures either against a standard or against other forms of the illusion. Typically, this procedure is used in attempts to demonstrate the existence of an illusion, and thus it is rarely used to measure the magnitude of the distortion as a function of various stimulus attributes. An example of this method is found in Bolles (1969), who simply asks $\mathrm{Ss}$ to judge the apparently longer section of the Mueller-Lyer against the apparently shorter in order to ascertain whether the illusion still exists when the configuration is of subfoveal size. One of the problems with paired comparison methods is the large number of judgments (and hence exposures) needed to obtain accurate data. Since many illusions are known to decrement with repeated exposures (Coren \& Girgus, 1972), one is apt to get underestimations of the illusory effect using these techniques.

A technique that seems to embody some of the advantages of the method of average error but that requires less in the way of apparatus involves the use of a graded series of comparison stimuli. The S's task is to select the comparison stimulus that most closely matches the test stimulus along the critical dimension. This method closely resembles a recognition or multiple choice paradigm, since the S simply looks over the simultaneously visible array until he finds the comparison figure that he feels is most similar to the test element. This procedure was used in $7 \%$ of the studies sampled. An example comes from Virsu (1971), who used the procedure to measure a curvature illusion by asking $S$ to select a circle from a graded series of circles, all visible simultaneously. The selected circle was to look like a continuation of the degree of curvature of a previously viewed arc. One of the problems with this type of measurement lies in the fact that $\mathrm{Ss}$ tend to select items from the middle of the series. There is also a propensity to select the same stimulus repeatedly in order to maintain apparent consistency. Ease of presentation and adaptability for group presentations are obvious advantages of this method.

Two subjective measurement techniques, which allow relative judgments but which, unfortunately, do not allow translation of responses into a physical metric, also appear in the sample. The first is a rating scale technique that asks $S$ to choose a verbal label that most clearly describes the figure that he is judging. This technique accounts for $8 \%$ of the studies reviewed. Restle and Merryman (1968) use this procedure to measure the magnitude of the Baldwin illusion (a size contrast figure). Ss are required to indicate whether the stimulus that they are viewing is "very long, long, moderately long, moderately short, short, or very short." The second subjective measure found in the sample is the method of magnitude estimation. This technique simply requires $S$ to assign a number proportional to the perceived size, curvature, etc., of the critical element in the illusion figure. This technique, 


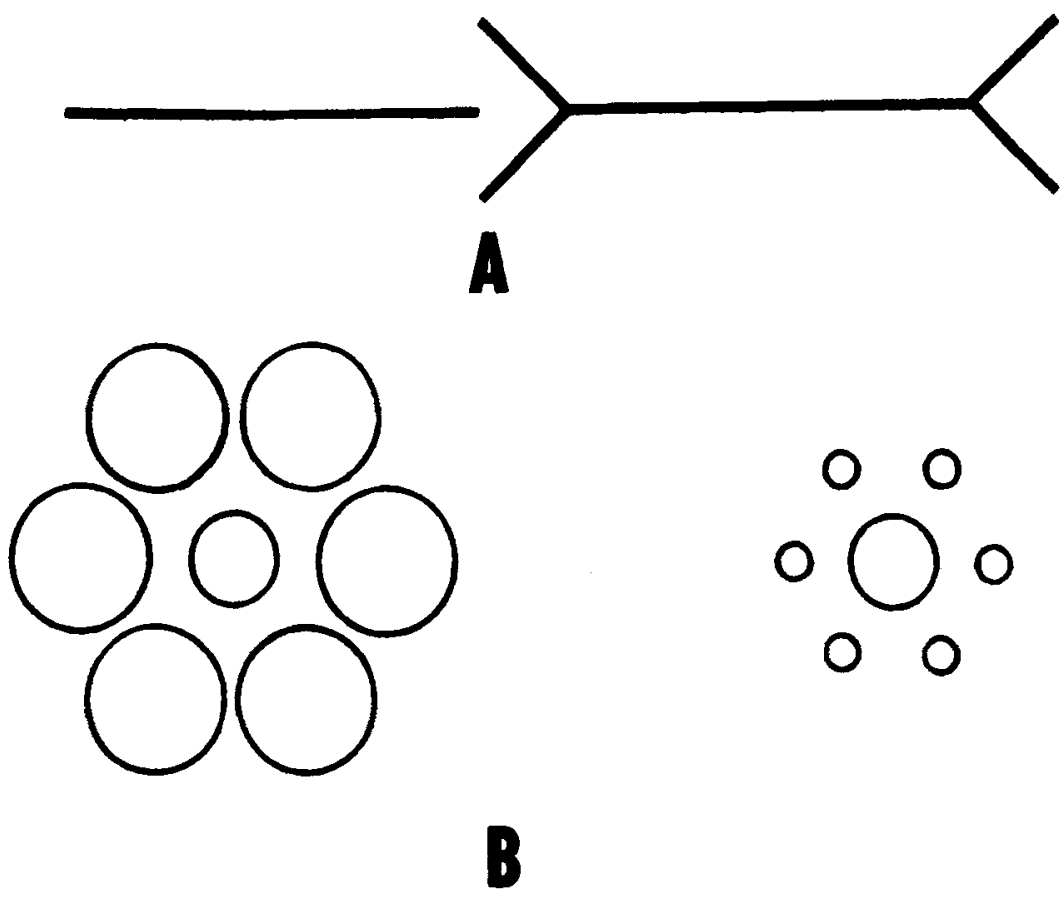

Fig. 1. A-The Mueller-Lyer configuration in which both horizontal lines are of equal length, although the line with the outgoing wings is apparently longer. B-The Ebbinghaus illusion in which both central circles are equal in size although the one surrounded by the larger circles is apparently smaller than the one surrounded by the smaller circles.

used either with or without a standard, accounts for $7 \%$ of the studies. An example of the use of this method is provided by Virsu (1967), who measured the magnitude of the Mueller-Lyer, the filled space-open space illusion, a perspective illusion and a variant of the Ebbinghaus illusion. Ss were simply required to estimate the apparent size of the critical elements in centimeters. Both of these techniques can be used in group paradigms and require little apparatus.

The remainder of the sample consisted of one crossmodal matching study (Moses \& Disisto, 1970) and a number of demonstrational papers that involved no direct measurements and account for the final $10 \%$. It is of interest to note that only $9 \%$ of the studies surveyed tested more than one $S$ per session, despite the fact that $44 \%$ of the studies sampled used methods that could easily be adapted to group presentation. This is probably due, at least in part, to a general suspicion about the adequacy and accuracy of group measures of illusion magnitude.

Given the variety of methods of illusion measurement found in this sample of the literature, it might be somewhat difficult for an investigator to decide among them, especially given the fact that few studies exist that test the relative efficiency of the various methods under comparable conditions and with comparable stimuli. In order to provide such a basis for choice, it seemed desirable to study systematically the sensitivity and efficacy of various measurement techniques in assessing parametric differences in illusion magnitude. As a secondary goal, it seemed desirable to assess whether measures of illusion strength suitable for group administration are presently available.

\section{METHODS Subjects}

Twenty-six undergraduate volunteers, participating for point credits in introductory psychology, served as Ss.

\section{Stimuli}

Since we are attempting to assess the usefulness of various methods used in parametric studies of illusion magnitude, two illusions whose magnitude can be varied systematically were selected: the Mueller-Lyer, which has long been known to vary in strength as a function of the angle between the wings (Lewis, 1909; Dewar, 1967) and the Ebbinghaus illusion (frequently called Titchener's circles), in which the apparent size of a central circle varies as a function of the size of a number of surrounding or context circles (Girgus, Coren, \& Agdern, 1972; Massaro \& Anderson, 1971). These configurations are shown in Fig. 1.

For the Mueller-Lyer, the apparently longer half of the configuration was used as a test stimulus. The shaft was $8 \mathrm{~cm}$ long and the wings were $2 \mathrm{~cm}$ long. The angle between the wings was 15,45 , or 75 deg. For the Ebbinghaus figure, the central test stimulus was an outline circle $14 \mathrm{~mm}$ in diam. Four context circles were arranged around the central circle at a distance of $6 \mathrm{~mm}$. These context circles could have diameters of $5,10,18$, or $23 \mathrm{~mm}$.

\section{Procedure}

The methods of measurement to be evaluated ithis study are the method of average error (adjustment), reproduction, selection from a graded series, rating scale, and magnitude estimation. These five methods represent the procedures used in $89 \%$ of the studies surveyed above, excluding those that utilized no formal measurement procedures.

\section{Method of Average Error}

For the Mueller-Lyer figure, Ss were required to adjust a variable line until it matched the length of a line distorted by the illusion. The variable line was drawn in black ink and set in a heavy pasteboard tongue and groove arrangement. A scale affixed to the sliding portion of the apparatus allowed readings to be taken in millimeters.

For the Ebbinghaus illusion, judgments were made by rotating a wheel that presented single comparison circles (with no surrounding circles) ranging in diameter from 8.0 to $19.5 \mathrm{~mm}$ in steps of $0.5 \mathrm{~mm}$. The circles appeared one at a time in a $26-\mathrm{mm}$ aperture cut into the apparatus.

\section{Reproduction Method}

For the Mueller-Lyer, the stimuli were printed one to a page. On each page, a 12-cm line was placed $10 \mathrm{~cm}$ down and somewhat to the right of the stimulus. $\mathbf{S}$ was required to estimate the apparent length of the shaft of the illusion by marking off the appropriate distance on this line. The procedure was similar for the Ebbinghaus figures, only here $\mathbf{S}$ was asked to indicate the apparent diameter of the test circle by marking off the appropriate length on the line.

\section{Graded Series Method}

Again, all test stimuli were printed one to a page. For the Mueller-Lyer, the set of comparison stimuli consisted of 27 line lengths (numbered 1 to 27) that ranged in size from 5.6 to $10.6 \mathrm{~cm}$ in $2-\mathrm{mm}$ steps. The comparison stimuli were presented in an ordered array on a separate page, with the shortest line at the top of the page and the longest at the bottom. Ss 


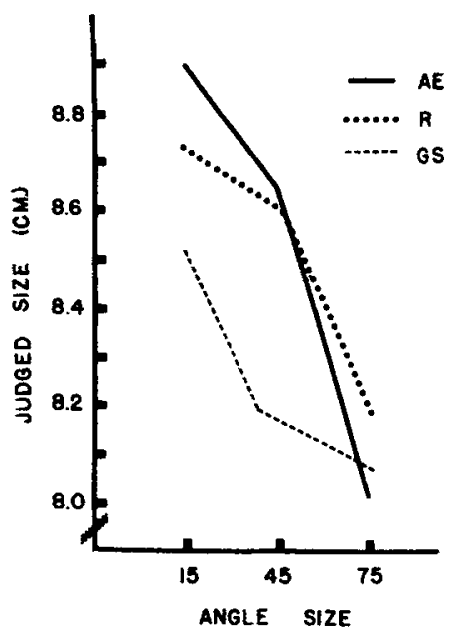

A

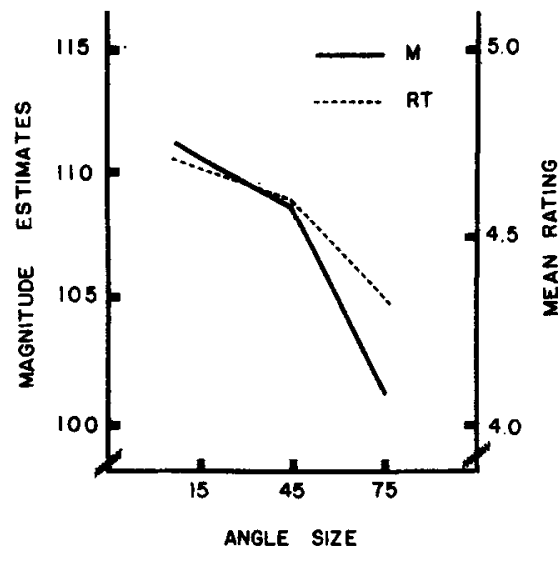

B

Fig. 2. A-The apparent size of the shaft of the Mueller-Lyer illusion in centimeters plotted as a function of angle size for the methods of average error (AE), reproduction (R), and selection from a graded series (GS). B-The relative size of the shaft of the Mueller-Lyer judged against a standard line measured via magnitude estimation (M) and rating scale (RT).

were asked to remove this page from their booklets so that it could be viewed easily at the same time as the illusion stimuli. They were required to judge which line most closely approximated the length of the shaft of the illusion figure. For the Ebbinghaus illusion, the graded series consisted of 17 circles, numbered from 1 to 17 and arranged in a similar graded series, with the smallest circle at the top of the page and the largest at the bottom. The circles ranged in size from 11 to $18 \mathrm{~mm}$ in steps of $0.5 \mathrm{~mm}$. Ss were required to indicate the number of the circle that most closely approximated the size of the test circle.

\section{Rating Scale}

For the Mueller-Lyer, in addition to the single illusion stimulus on each page, $10 \mathrm{~cm}$ down and to the right of the figure there appeared an $8 \mathrm{~cm}$ line (equal in length to the shaft). At the bottom of the page there was a 7-point rating scale labeled "very much larger, much larger, larger, equal, smaller, much smaller, very much smaller." Ss were required to check the point on the scale appropriate to their perception of the relative size of the illusion test element compared to the standard. A similar procedure was followed for the Ebbinghaus figure, only here the standard was a 14-mm-diam outline circle.

\section{Magnitude Estimation}

As in the rating scale procedure, each Mueller-Lyer stimulus appeared on the same page as an $8-\mathrm{cm}$ standard, only now the standard was labeled are manifested; (2) whether the obtained results are stable enough to yield statistically significant effects as a result of the parametric manipulations; and (3) the nature of the relationship between the magnitude of the observed effects and the variability of measurement. We will consider each of these points in turn.

The first point concerns whether each of the methods produces the expected results. For the apparently longer segment of the Mueller-Lyer, the data of Lewis (1909) would lead us to expect that the apparent length of the shaft will decrease as the angle between the wings increases. For the Ebbinghaus figure, the extant data lead us to expect that the perceived size of the central test circle will vary inversely with the size of the surrounding circles.

Unfortunately the data cannot all be presented on any one graph, since it is impossible to convert all of the data to any single metric. Although the method of average error, reproduction, and selection from a graded series all allow the direct translation of judgments into physical equivalents, the rating scale and method of magnitude estimation do not. For this reason, the data from the former three methods will be presented on one graph while the data from the latter two methods will be plotted on a separate graph. Figures $2 a$ and $2 b$ present the data from the Mueller-Lyer, and Fig. $3 a$ and $3 b$ contain the data from the Ebbinghaus figure.

It is clear from the figures that the expected ordinal relationships do appear in all of the measurement methods tested. All five methods show a decrease in apparent size of the Mueller-Lyer shaft with larger angles between the wings. Similarly, the apparent size of the central test circle in the Ebbinghaus configuration varies inversely with size of the inducing circles. The only perturbations appear in the Ebbinghaus figure where the methods of selection from a graded series and magnitude estimation fail to show a difference between the 18- and 23-mm inducers.

It is interesting to note that all of the curves for the Ebbinghaus figure show underestimation relative to the actual physical size of $14 \mathrm{~mm}$. Thus, although the expected relative size changes appeared in the data, the usual absolute changes did not. However, as pointed out by Massaro and Anderson (1971) and Girgus et al (1972), this probably indicates a constant error that is due to contextual effects operating on the comparison circles as well as on the test circles. These effects do illustrate that in situations 


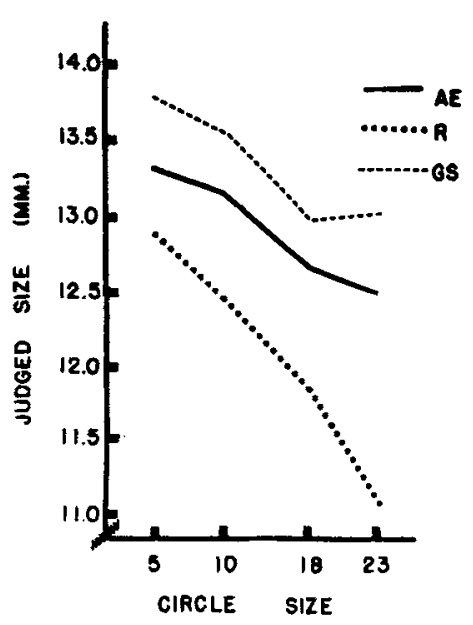

A

Fig. 3. A-The apparent size of the central test circle in the Ebbinghaus illusion in millimeters plotted as a function of the size of the surrounding circles in millimeters for the methods of average error ( $A E)$, reproduction (R), and selection from a graded series (GS). B-The relative size of the central circle judged against a standard circle measured via magnitude estimation (M) and rating scale (RT).

where absolute measurements are needed (rather than relative illusion magnitudes), a control figure with no inducing components should be included to provide a baseline.

The statistical results do not show as consistent a pattern as do the figures. Table 1 presents the appropriate comparisons. The column marked " $F$ " indicates the $F$ ratios computed from analyses of variance performed on the data from each illusion and each measure. These represent the main effect of angle size for the Mueller-Lyer and of inducer size for the Ebbinghaus figure.

For the Mueller-Lyer, all of the methods show the expected variation of shaft size to be significant at $\mathrm{p}<.05$ or better, except for the rating scale data. For the Ebbinghaus configuration, all of the methods show statistical reliability at the $5 \%$ level or better except for the magnitude estimation task. The reasons for this relative insensitivity of the subjective scales are not immediately apparent. Certainly it is not the case that Ss did not understand the task, since all those interviewed after the sessions reported no difficulty whatsoever. It is possible that experimentally naive Ss may need some practice with these subjective procedures in order to establish some frame of reference or internal modulus. This is supported by the fact that Restle and Merryman (1968), when using a rating scale to measure the magnitude of the Baldwin illusion, felt it necessary to provide a practice series of judgments on the entire set of stimuli, before collecting his data. Following this practice run, fairly stable data were obtained. A more direct test of the stability of subjective

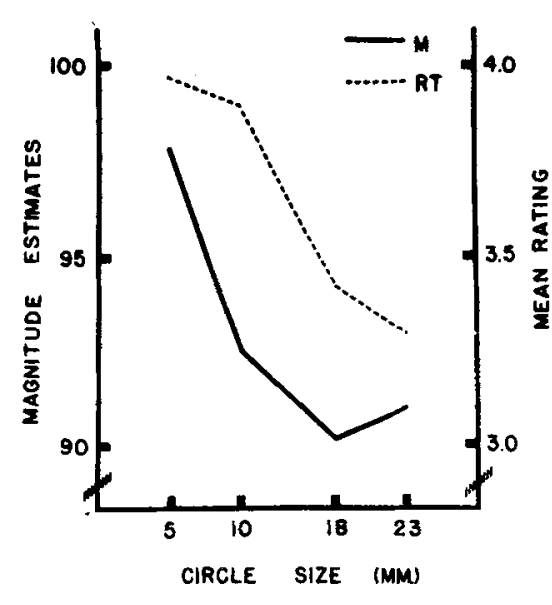

B squares of the means, over the total sum of squares, with both values corrected for the magnitude of the error variance (Hays, 1963). The column marked $\omega^{2}$ in Table 1 contains these values. For the Mueller-Lyer, the indication is that the method of average error demonstrates a very strong effect, producing results in which the variation of angles explains some $23.3 \%$ of the variance. The method of reproduction and magnitude estimation are only one-third as efficient, handling some $7 \%$ of the variance, and the graded series explains only $3 \%$. (Note that, for nonsignificant $F$ ratios such as that manifested by the rating scale data, $\omega^{2}$ is automatically set to zero.) For the Ebbinghaus figures, the graded series is actually the most efficient, with $18 \%$ of the variance explained by size of the inducer, and the method of average error and rating scale are equivalent to each other, with $14 \%$ of the variance explained.

Some conclusions and recommendations can be drawn from these data and their analysis. First, it must be made clear that the observers who participated in this experiment were experimentally naive and made only one judgment per figure per measure. Thus, we are utilizing a very stringent set of conditions to measure the effectiveness of the techniques. The fact that four out of five measures produced the expected results at acceptable levels of statistical reliability for each illusion is heartening. The failure of one of the subjective measures to demonstrate reliable changes in illusion magnitudes for each of the illusion figures is distressing but may reflect the necessity for pretraining before the actual measurement session rather than any inherent weakness in these scaling procedures. Note, however, that any such pretraining may introduce the problem of illusion decrement. In addition, the fact that the subjective techniques of rating scale and magnitude estimation do not provide a means of translation of the data back into corresponding physical units renders it difficult to compare results of studies conducted under differing conditions or in different laboratories.

Since the methods of average error,

Table 1

Statistical Tests and Proportion of Variance Explained by Each Measure

\begin{tabular}{|c|c|c|c|c|}
\hline \multirow[b]{2}{*}{ Measure } & \multicolumn{2}{|c|}{ Mueller-Lyer } & \multicolumn{2}{|c|}{ Ebbinghaus } \\
\hline & $\mathbf{F}$ & $\omega^{2}$ & $\mathbf{F}$ & $\omega^{2}$ \\
\hline $\begin{array}{l}\text { Average Error } \\
\text { Reproduction } \\
\text { Graded Series } \\
\text { Rating Scale } \\
\text { Magnitude Estimation } \\
\text { Critical Value for } p<.05\end{array}$ & $\begin{array}{r}\mathbf{3 1 . 4 5} \\
\mathbf{8 . 4 3} \\
\mathbf{3 . 8 3} \\
1.39 \\
4.04 \\
\mathbf{3 . 1 5}\end{array}$ & $\begin{array}{l}0.23 \\
0.07 \\
0.03 \\
0.00 \\
0.07 \\
-\end{array}$ & $\begin{array}{r}9.72 \\
8.43 \\
12.24 \\
8.14 \\
0.61 \\
2.76\end{array}$ & $\begin{array}{l}0.14 \\
0.03 \\
0.19 \\
0.14 \\
0.00 \\
-\end{array}$ \\
\hline
\end{tabular}


reproduction, and selection from a graded series produced statistically reliable results for both configurations, one would be tempted to designate them as the methods of choice. The expected, overwhelming superiority of the method of average error, based upon its traditional use whenever possible, even in the face of complicated apparatus difficulties, did not emerge. Under the conditions tested, the methods of reproduction or selection from a graded series seem to give nearly equivalent results to the method of adjustment. These latter two methods have the additional advantages of requiring little apparatus and being suitable for group testing. The fact that these data were actually gathered in a group testing situation indicates that the traditional distrust of group measures may be unwarranted, at least if the appropriate measurement techniques are employed.

In sum then, we can conclude that the methods of average error, reproduction, and selection from a graded series are efficient measures of variations in illusion magnitude, and, at least under conditions where no pretraining is available to the observers, these measures are probably preferable to rating scales or the method of magnitude estimation.

REFERENCES

BOLLES, R. C. The role of eye movements in the Müller-Lyer illusion. Perception \& Psychophysics, 1969, 6, 175-176.

BURNHAM, C. A. Decrement of the Muller-Lyer illusion with saccadic and tracking eye movements. Perception \& Psychophysics, 1968, 3, 424-426.

COREN, $S$, The influence of optical aberrations on the magnitude of the Poggendorff illusion. Perception \& Psychophysics, $1969,6,185-186$.

COREN, $S$. Lateral inhibition and the Wundt-Hering illusion. Psychonomic Science, 1970a, 18, 341.

COREN, $\mathbf{s}$. Lateral inhibition and geometric illusions. Quarterly Journal of Experimental Psychology, 1970b, 22, 274-278.

COREN, S., \& GIRGUS, J. S. Illusion decrement in intersecting line figures. Psychonomic Science, 1972, 26, 108-110.

DEWAR, R. E. Stimulus determinants of the magnitude of the Mibller-Lyer illusion. Perceptual \& Motor Skills, 1967, 24, 708-710.

ERLEBACHER, A., \& SEKULER, $R$. Explanation of the MüllemLyer illusion: Confusion theory examined. Journal of Experimental Psychology, 1969, 80, 462-467.

EYMAN, R. K. The effect of sophistication on ratio and discriminative scales. American Joumal of Psychology, 1967. 80, 520-540.

FESTINGER, L., WHITE, C. W., \& ALLYN, M. R. Eye movements and decrement in the Muller-yyer illusion. Perception \& Psychophysics, 1968, 3, 376-382.

GIRGUS, J. S., COREN, S., \& AGDERN, M. The interrelationship between the Ebbinghaus and Delboeuf illusions. Journal of Experimental Psychology, 1972, in press.

HAYS, W. L. Statistics. New York: Holt, Rinehart \& Winston, 1963.

JUDD, C. H. A study of geometrical illusions. Psychological Review, 1899, 6, 241-261.

JUDD, C. H. Practice and its effects on the perception of illusions. Psychological Review, 1902, 9, 27-39.
JUDD, $C$. H. The Müller-Lyer illusion. Psychological Review, Monograph Supplement, 1905, 7, (Whole No. 29) 5581.

LEWIS, E. O. The effect of practice on the perception of the Mutller-Lyer illusion. British Journal of Psychology, 1908, 2 , 294-306.

LEWIS, E. O. Confluxion and contrast effects in the Müller-Lyer illusion. British Journal of Psychology, 1909, 3, 21-41.

MASSARO, D. W., \& ANDERSON, N. H. Judgmental model of the Ebbinghaus illusion. Journal of Experimental Psychology, 1971, 89, 147-151.

MOSES, F. L., \& DesISTO $M$ J Arm-movement responses to Müller-Lyer stimuli. Perception \& Psychophysics, $1970,8,376-378$.

PRESSEY, A. W., \& SWEENEY, o. A variation of the Poggendorff illusion. Perceptual \& Motor Skills, 1969. 28, 883-886.

RESTLE, F., \& MERRYMAN, C. T. An adaptation-level theory account of 8 relative-size illusion. Psychonomic Science, 1968, 12, 229-230.

SEASHORE, C. E., CARTER, E. A. FARNUM, E. C." \& SIES, R. W. The effect of practice on normal illusions. Psychological Review, Monograph Supplement, 1908, 9, 103-148.

SEASHORE, C. E. \& WILLIAMS, M. C. An illusion of length. Psychological Review $1900,7,592-599$.

SEASHORE, C. E., \& WTLLIAMS, M. C. An illusion of length. University of Iow Studies in Psychology, 1902, 3, 29-37.

VIRSU, V. Geometric illusions, I. Effects of figure type, instruction, and pre- and intertrial training on magnitude and decrement of illusion. Scandinavian Journal of Psychology, 1967, 8, 161-171.

VIRSU, $V$. Underestimation of curvature and task dependence in visual perception of form. Perception \& Psychophysics, $1971,9,339-342$. 